\title{
Nursing Students' Clinical Practice Experience During the Internship Year at Different Hospitals: A Qualitative Study
}

\author{
Samar Sultan Althaqafi", Hala Abdullah Alhatimi*, Alhanouf Meteb Almalki, \\ Ola Muneer Alyamani, Maram Ahmed Banakhar
}

Department of Public Health, Faculty of Nursing, King Abdulaziz University, Jeddah, Saudi Arabia

Email address:

Althaqafisamar@gmail.com (S. S. Althaqafi),Alshreefhala@gmail.com (H. A. Alhatimi)

${ }^{*}$ Corresponding author

\section{To cite this article:}

Samar Sultan Althaqafi, Hala Abdullah Alhatimi, Alhanouf Meteb Almalki, Ola Muneer Alyamani, Maram Ahmed Banakhar. Nursing Students' Clinical Practice Experience During the Internship Year at Different Hospitals: A Qualitative Study. American Journal of Nursing Science. Vol. 8, No. 5, 2019, pp. 255-262. doi: 10.11648/j.ajns.20190805.18

Received: August 1, 2019; Accepted: September 10, 2019; Published: September 26, 2019

\begin{abstract}
Background: An internship programme is an important period that enables nursing students to work and function as primary nurses, with three to eight patients per shift, in order to develop their clinical skills by integrating theory with practice and improving their assessment, problem solving, and interpersonal skills. Having undergraduate nursing interns from different colleges in one clinical placement may explain the variation regarding the factors, challenges, and barriers that affect students' clinical practice during the internship period. Study aim: This study aimed to explore the clinical practice experiences of nursing students doing internship years at different hospitals in Saudi Arabia. Method: A descriptive qualitative study was conducted with nursing interns from the Faculty of Nursing at a university in Saudi Arabia, who were doing internship years at different hospitals. The study participants were selected using purposive sampling. The data were collected via three semistructured focus group interviews and analyzed using thematic analysis. Results: Educational programmes, hospital orientation programmes, and mentorship programmes were all highlighted as the most important influencing factors in the interns' clinical practice. In addition, unfair treatment, the ignorance of healthcare professionals, and being involved in non-nursing work were highlighted as challenging factors that negatively affected the interns' clinical practice. Conclusion: There is a need for continuous educational programmes and mentorship programmes to be provided for all nursing interns, in particular by hospital education departments. In addition, a clear job description and list of responsibilities for all nursing interns should be provided at the start of the internship programme in order to prevent interns from being involved in non-nursing work.
\end{abstract}

Keywords: Nursing Students, Internship Programme, Clinical Practice, Challenges in Clinical Practice

\section{Introduction}

Nursing programmes in Saudi Arabia offer a four-year baccalaureate degree that consists of three years of theoretical education followed by an internship year, which consists of forty-seven weeks of clinical practice in a hospital setting with the direct supervision of the college. This internship period is a mandatory requirement of the Saudi Council for Health Specialties in Saudi Arabia for practising nursing formally [1]. The internship programme is defined as "one-to-one experience with a registered nurse $(\mathrm{RN})$ at his/her place of employment that is time limited and has faculty mentor support" [2]. It is an important period that allows nursing students to work and function as a primary nurse, with three to eight patients per shift, in order to develop their clinical skills by integrating theory with practice and improving their assessment, problem solving, and interpersonal skills [3]. Furthermore, the internship programme enables nursing students to manage the workload of having many patients requiring full patient care, enhance their clinical skills, set priorities, and gain experience in implementing nursing processes. Moreover, through the internship programme, an expert nurse is given the opportunity to play an active and major role in teaching, enhancing critical thinking, and guiding and assisting the students under his/her supervision [2].

Previous research has identified several factors that 
influence undergraduate nursing students' experiences during their internship period. For instance, one study sought to explore the factors affecting the clinical practice of nursing interns at a tertiary hospital and found that the students had a positive experience [4]. They developed their communication, leadership, and coping skills and the internship programme provided deeper insight into the nursing profession that helped them make informed career decisions [4]. Another study explored why nursing students find it difficult to turn taught theoretical knowledge into clinical practice [5]. The interviews conducted in the study were analyzed using a constant comparative method and revealed that although the quality of academic nursing curriculums was perceived by participants as increasing, clinical behaviours in both the practice settings and education were perceived as "traditional routine-based". Furthermore, the mentoring of nursing interns by preceptors was identified as an important aspect of educating, supporting, and supervising the interns in their clinical practice. Additionally, nursing intern mentors were not always compensated for their work [2].

In Saudi Arabia, there are twenty-five governmental nursing colleges and twenty-two private nursing colleges that offer a baccalaureate degree in nursing science, which includes a compulsory internship period [1]. However, the rules and regulations of the internship programmes differ from one college to another, which can pose some challenges for both students and healthcare organizations. According to the internship programme, nursing interns from all nursing colleges in Saudi Arabia are usually distributed among different healthcare organizations. Having student nurse interns from different colleges in one clinical placement may explain the variation regarding the factors, challenges, and barriers that affect undergraduate nursing students' clinical practice during the internship period.

The internship period is compulsory for undergraduate nursing students in all nursing programmes at different universities in Saudi Arabia. The aim of the internship period is to educate nursing students in general clinical practice so they can gain the necessary knowledge and skills for general nursing practice and develop appropriate nursing attitudes and ethics in accordance with the teachings of Islam. Therefore, providing a well-planned internship programme allows nursing students to effectively develop different clinical skills, integrate theory with practice, and develop problem solving and interpersonal skills [5]. In addition, well-organized internship programmes enable nursing students to become familiarized with the formal and informal norms, protocols, and expectations of the nursing profession and the healthcare systems [6]. In this way, the internship programme plays an important role in preparing all nursing students for working and acting as professional nurses. However, nursing interns experience different factors and challenges in different healthcare organizations at different hospitals, which research has shown can affect their ability to work effectively and to be sufficiently confident at the end of the internship programme. This has several consequences, such as making them feel unprepared to work as a professional nurse or as a staff in future. Most importantly, they may feel incompetent in performing different clinical procedures, resulting in patient harm. However, few international studies have explored the different factors and challenges affecting experiences of nursing interns. Indeed, the authors of the current paper were able to locate just one study that explored the factors and challenges encountered by undergraduate nursing students in Saudi Arabia during the internship period, and it was limited to one tertiary hospital. This revealed a gap in the literature regarding the experiences of student nurse interns located in different healthcare organizations in Saudi Arabia and the potential to improve the internship programme and to better prepare nursing students for selecting the best clinical placement in the future. Therefore, this study aimed to explore the clinical practice experiences of student nurses doing their internship year at different Saudi hospitals.

Several studies have explored the factors affecting nursing interns' clinical practice in different clinical placements outside of Saudi Arabia. For instance, an explorative descriptive qualitative study was conducted in Ghana that explored thirty-five undergraduate nursing students' experiences in two teaching hospitals [7]. The data were collected via semi-structured interviews, and the findings of the study revealed that the majority of the undergraduate nursing students identified different factors that affected their clinical practice, some of which they highlighted as negative experiences and challenges, such as feeling isolated, experiencing poor application of the nursing procedures, and poor examples of physical examinations given by the staff nurses. The results of the research also demonstrated that the undergraduate students used complicated medical devices and were given the opportunity to observe various complex medical conditions and cases, which they considered a positive experience. Finally, the participants demanded support from different level of supervisory obligation and logistics provisions to learn skills related to physical examinations and nursing procedures during clinical placements [7].

Similar results were demonstrated in the qualitative study conducted in Saudi Arabia that aimed to explore the factors affecting nursing interns' clinical practice [4]. Data were collected via interviews with undergraduate nursing students doing their internship programme at a tertiary hospital. The study participants identified different positive and negative factors affecting the internship programme, and also highlighted the different nursing interns' unique learning requirements. The majority of the respondents perceived their clinical experience as a poor learning environment as they felt they were treated unfairly by their instructors. The nursing interns described being ignored by the staff, feeling embarrassed, and feeling restricted as negative factors. However, they also identified positive factors that enhanced their clinical experience, such as the development of personal growth and leadership skills which helped them become more independent in their practice and develop good 
communication skills for dealing with different kinds of people. Finally, the interns perceived the internship programme as an ideal chance for students to reflect on their learning [4].

Contrary to the above, a recent qualitative descriptive study was carried out to explore Saudi male nurses' clinical practice experiences using an inductive content-analysis approach to analyze the data [8]. This study investigated the experiences of twenty-two final year male nursing students from three public hospitals in a main city, and identified three major challenges faced by the participants during their internship year. The results indicated that the negative societal and cultural image of male nurses, the lack of male students' engagement in nursing practice, and the lack of programme policies, rules, and regulations regarding personal and cultural needs were the main challenges contributing to increased burnout in the nursing profession and negatively affecting male students' self-esteem [8]. Likewise, a descriptive cross-sectional study was conducted among 300 Saudi nursing interns to validate and measures cultural adapt the Arabic version of self-efficacy for clinical evaluation scale (SECE) and explore nursing interns' perceived confidence. The study found that the Arabic version of SECS was successfully cross-culturally adapted to reveal that gender, duration of internship, and place of training affected the perceptions of the Saudi nursing interns regarding self-efficacy [9]. In this way, the instrument could help preceptors formulate more appropriate strategies for enhancing nursing intern competence and promoting their self-efficacy as they transition to the role of registered nurse [9].

With regard to the clinical competence and preceptorship aspects of the internship, a mixed-method research study was carried out in a tertiary hospital, which utilized a descriptive cross-sectional approach to explore the factors impacting the perception of nursing interns regarding clinical competence [10]. The study aimed to determine whether the preceptorship programme provided the participants with the necessary education for enhancing their clinical competency. Data were collected from ninety-two undergraduate nursing interns who had completed five years in nursing education, including the preceptorship programme, using a survey questionnaire that focused on competency and the improvement of the internship programme. The study revealed that the preceptorship programme promoted the interns' competencies, primarily the competencies of prioritizing acutely ill patients, demonstrating complex nursing skills, and multi-tasking. The majority of the participants perceived the preceptorship as a constructive experience and the availability, approachable attitude, trustworthiness and reliability of the preceptor as influencing factors in enhancing their clinical competence. Significant differences were observed related to internship stage and type of school. Moreover, the results also revealed a positive relationship between the clinical competencies in general performance and nursing interns' efficacy, professional behaviour, and fundamental nursing skills. It was clear that the preceptorship programme positively influenced the nursing interns' ability to deal with actual patients in clinical settings. Thus, the study highlighted the need for preceptorship programmes to teach techniques that advance junior nurses to the next stage of clinical competence [10].

\section{Methods}

\subsection{Design}

A descriptive qualitative research design was used in this study to explore nursing students' clinical practice experiences during the internship period as well as the challenges encountered by working in different hospitals. Qualitative research is frequently used to describe and understand human behaviour or experiences related to a particular phenomenon [11]. Qualitative research draws on subjective data that is difficult to code numerically and focuses on feelings, experiences, and the content of people's articulations [12]. Therefore, in-depth information and understanding regarding a phenomenon is best revealed using qualitative methods as they provide answers to 'how' and 'why' type questions, such as how nursing students perceive and encounter clinical practice during internships at different hospitals.

\subsection{Settings}

This study was conducted with nursing students from the Faculty of Nursing at one selected university in Saudi Arabia who were doing their internship year at different hospitals. The Faculty of Nursing at the selected university offered both graduate and postgraduate programmes. The graduate programme was comprised of a foundation year and a threeyear baccalaureate programme in nursing science followed by a one-year internship programme. The nursing students were allowed to do their internship year at any governmental hospital in the Kingdom of Saudi Arabia. The total number of nursing interns in the Faculty of Nursing at the university was eighty-seven.

\subsection{Sampling and Sample Size}

The sample of this study consisted of bachelor's nursing students at a university in Jeddah city who were doing the internship programme at different hospitals. The reason for targeting nursing interns was based on the literature review conducted for this study, which revealed that nursing interns experience different issues and challenges that affect their clinical practice during the internship programme. Purposive sampling was used to recruit the nursing intern participants. Purposive sampling seeks to select a study sample that can provide 'information-rich cases' in order to explore the significant issues related to the study phenomenon [12].

Qualitative studies rely on a small number of participants with the aim of achieving an in-depth investigation. The sample size of this study was based on data saturation. Three focus group interviews were conducted with a total of twelve participants from different hospitals who were recruited 
according to the following inclusion criteria: (1) All nursing students must be bachelor's students currently doing their internship year; (2) Any nursing intern who is working a 12hour shift. Nursing interns on the bridging programme were excluded from the study.

\subsection{Ethical Considerations}

\subsubsection{Ethical Approval}

Ethical approval for this study was obtained from the Faculty of Nursing at the selected university in Saudi Arabia.

\subsubsection{Anonymity and Confidentiality}

Ethical principles regarding the protection of privacy and avoidance of deception were followed in this study [12]. The participants' names and identity were not revealed during the process of data collection and analysis [11]. The participant information sheet provided all the required information regarding the study and voluntary participation to the participants. In addition, the participants' names were removed and each respondent was ascribed a code during the data analysis and reporting of the findings in order to protect their identity.

\subsubsection{Informed Consent}

The participant information sheet was written clearly to explain the purpose of the research study. In addition, the participant's right to refuse to participate in the study without giving a reason was clearly stated. All the information necessary for making an informed decision regarding involvement in the study were provided to the study participants. Prior to starting the data collection, the participants were given the opportunity to ask any questions. All participants who agreed to participate in the current study completed and signed the consent form.

\subsection{Data Collection Method}

The data were collected via semi-structured focus group interviews with the nursing interns. The aim of conducting these focus groups was to describe, explore, and explain the nursing interns' experiences, views, and beliefs regarding the internship programme. Furthermore, the focus group interviews had the advantage of helping nursing interns to reflect on their own experiences as part of a group [13]. In addition, the dynamic interaction between group members provided additional data and suggestions regarding how to improve and enhance the internship programme which would not have arisen from individual interviews [14]. The interview framework for this study was designed to address areas and themes related to the research questions according to the literature review. Hence, the focus group interviews investigated which factors affected the nursing interns' clinical practice, which factors eased and facilitated the clinical practice of the nursing students during the internship programme, and the issues and challenges faced by the nursing interns doing the internship programme at different hospitals.

\subsection{Data Collection Process/Procedure}

After obtaining ethical approval from the Faculty of Nursing, the list of all nursing interns in the faculty was used to contact potential participants and recruit them into the study. The individuals who met the inclusion criteria were approached during the monthly meeting between the nursing interns and the internship supervisor at the Faculty of Nursing. In the meeting, the researchers introduced the research topic and provided all the required information to all the potential participants. All individuals who were willing to participate were contacted and notified of the focus groups that would be held at the Faculty of Nursing. Three focus group interviews were conducted for the study, all of which were audio recorded and lasted for one hour. The data collection was conducted from February to March 2019.

\subsection{Data Analysis}

The data that emerged from the interviews was analyzed using thematic analysis, which was conducted via the following steps: first, the researchers transcribed verbatim all the recorded interviews; secondly, the researchers read all the transcripts to ensure they were familiar with the data; third, the first level codes were developed from each interview transcript; fourth, the researchers reviewed all the first level codes to generate second level codes (sub-categories); and finally, the researchers identified and refined the emergent themes [12].

\section{Results}

The focus groups interviews yielded a large quantity of information regarding the internship programme, despite the fact that the interns' reported experiences varied significantly according to which hospital they worked in. This demonstrated that the internship programme was affected by several factors and barriers within two major themes that emerged from the analysis of the focus group data.

\subsection{Factors Influencing Clinical Practice During the Internship Programme}

This theme highlighted five main factors affecting the nursing interns' clinical practice: the support of the nursing staff, the hospital orientation programme, the preceptorship programme, educational programmes, and the responsibility level of the nursing interns.

\subsubsection{Support of the Nursing Staff}

All participants from the different hospitals agreed that the nursing staff were very supportive. For example, they encouraged the interns to attend teaching sessions and gave them the opportunity to participate in nursing procedures. Moreover, they were available to answer the interns' questions and requirements: "The nurse encouraged me to not be shy and asked if I had any concerns or if I needed anything. I told her that I just wanted her to teach me how to detect the right vein. She gave her work to another nurse and 
came to teach me" -(HM).

\subsubsection{Hospital Orientation Programme}

All the participants described attending a general orientation programme at their hospital before starting their clinical practice, because it was crucial for them to be aware of hospital policies and procedures. However, the hospital orientation programme differed from one hospital to another. For example, a respondent who attended the military hospital explained that the orientation programme involved training regarding elements of the nursing care system such as nursing documentation: "Others were attending and profiting from it as much as possible by learning about the system of nursing documentation procedures" - (HM). Moreover, the interns described receiving three days' orientation training in the surgical and ICU units to better understand the unit's routine and nursing care system: "There are orientation days in the surgical unit and ICU. They are mostly three days, and you shadow the nurse preceptor or sometimes you help her with her patients" - (RM). A respondent from the teaching hospital said they received one week of hospital orientation presented by several clinical instructors who explained the policies and procedures of nursing practice, which was then followed by six competency exams: "It was one week of lectures about nursing procedures given by each clinical instructor from each unit in the hospital... We had six competency exams at the end of the orientation programme: medication administration, drug calculation, IV cannulation, $I V$ medication, blood transfusion, and $A B G^{\prime \prime}-(\mathrm{ST})$. On the other hand, the interns from the specialized research hospital reported taking a two-week hospital orientation programme incorporating medication calculation, safety, and blood transfusion. In addition, the interns highlighted some procedures that were given particular focus, such as IV cannulation, NGT, and medications: "We had a two-week general nursing orientation; they taught us about medication, safety and blood transfusion... Some procedures were given additional focus, such as IV cannulation, medication, NGT, and chest tubes" - (RS).

\subsubsection{Preceptorship Programme}

Preceptorship was perceived as an important factor for all interns, although it varied in each hospital, which affected the interns' ability to learn and practise. For instance, the interns from the military hospital stated that each intern was assigned a preceptor, but the preceptors only used the interns to assist them with patient care by taking vital signs and administering medications: "For each student nurse intern there is a nurse preceptor... Some of them don't want anything from you except to check vital signs and blood sugar, and some ask for help with their patients to help me with my patients." - (RM). In addition, the interns at the teaching hospital stated that they felt confident in providing independent nursing care and only used the preceptors to gain access to the system: "We have one, but I hate that she is with us the entire internship year, I feel confident and independent now but the preceptor is still with me, which I find annoying, I only need her to gain access to the system and when I get medications." -(ET). On other hand, the preceptorship programme at the specialized research hospital was perceived as very structured and effective by the nursing interns. For example, there was a different preceptor in each unit and her role was to assist the interns with practising safely and to educate them regarding the most common medications and their adverse effects. Furthermore, each preceptor orientated each nursing intern to the unit as well as to the scope of practice: "The preceptor helps us to practise in a safe way, she/he teaches me about the adverse effects of medications" -(RS); "Also, the preceptor does the orientation of the unit and tells us the bed capacity and common diseases and scope of practice" -(AS).

\subsubsection{Educational Programmes}

Several educational programmes were provided by each hospital that affected the interns' ability to acquire the required knowledge during the internship programme. In the military hospital, the interns attended educational programmes with the hospital staff, as there was no programme or project provided specifically for them: "I went to register and ask about the programme and they told me that it was only for nursing staff and if there was an available place they would contact me" - (HM). Similarly, the interns at the teaching hospital attended educational sessions "...sometimes with the nurses. We had one for needle stick injury that all the interns were required to attend" - (ST). Moreover, the interns had to present a case study at the end of the internship programme: "Each group has a case study to present at the end of the internship - each group from the different colleges" - (WT). However, the interns at the specialized research hospital stated that there were several educational programmes that were attended by interns and nursing staff to discuss new subjects monthly. In particular, they explained that an educational programme was provided for the nursing interns specifically that required them to attend a work-based learning day every three weeks to discuss all new cases and procedures: "Every three weeks we have a work-based learning day and it's very helpful. Sometimes we talk about new procedures or case studies and at the end we have an exam and I think it has prepared us for the SNLE exam" - (SS). The nursing interns at this hospital also had a workbook that included several checklists for different procedures applied in different units and contained several questions to research, which the participants found very helpful for updating their skills and knowledge: "The workbook contains our experimental checklists. It's like objectives, but not for every unit. It contains the steps for certain procedures and has a lot of questions that we research about to solve. A lot of these questions will come up in the SNLE exam, so it's very helpful in giving us skills and knowledge"- (SS). In addition, one of the advantages of this specialized research hospital identified by the participants was the mentorship programme offered to all interns at the beginning of the internship which provided support to interns regarding any issues they encountered and guided them during the internship programme. Hence, the respondents 
stated that they preferred to speak to their mentors more than their preceptors: "If you have a problem you can ask her/him what the best thing to do in this situation is, and also when we take the elective months, we can ask her/him about the objectives that we want to achieve" - (RS); "I feel like she is closer to us than the preceptor, because she has had the same experiences as me, she understands me" - (SS).

\subsubsection{The Responsibility Level of the Nursing Interns}

The level of responsibility given to the nursing interns varied according to each hospital's policy. For instance, in the teaching hospital, the nursing interns were assigned one to three patients within each rotation and given total responsibility for those patients' care. Similarly, in the military hospital the interns were assigned a maximum of three patients, and the majority of the assigned cases were DNR: "We are assigned one patient and from the third week to the end of rotation in a unit we are assigned two to three patients" - (WT); "DNR patients aren't considered a critical case, or are they? If they are, we are handling DNR cases in the medical unit" - (HM). In the specialized research hospital, the interns were assigned total responsibility for two patients: "I take care of one patient at the beginning for four months, then they decide if I'm confident and good enough and then they assign me two patients until the end of the internship program"- (AS). They were also allowed to do all procedures except for blood transfusions: "As interns we are still not allowed to do blood transfusions alone" - (SS); "It depends on the unit; for example, in the oncology unit, the post bone marrow transplant patients have low immunity, so we are not allowed to handle them" - (RS).

\subsection{Challenges Faced by the Nursing Interns During the Internship Programme}

This theme highlighted the challenges experienced by the nursing interns during their internship year, such as patient culture, the relationship between nursing interns and other healthcare professionals, non-nursing work, and time management.

\subsubsection{Patient Culture}

The nursing interns described encountering some difficulties regarding patients culture, especially in the military hospital. For example, the majority of the patients were not aware of the presence of Saudi nursing interns working at the hospital, which was perceived as a barrier to communicating with patients and providing safe nursing care: "One of my patients was talking to me like I'm not an Arabic nurse, and I tried to make her understand that I'm a Saudi, but she was insistent that I'm not Saudi. I think because they got used to nurses being from India or the Philippines" - (HM).

\subsubsection{The Relationship Between Nursing Interns and Other Healthcare Professionals}

The relationship between nursing interns and other healthcare professionals was perceived differently according to hospital. The interns at the military hospital reported receiving unfair treatment from some of the nursing staff, particularly during patient handovers, as a challenge: "...unfair treatment during endorsement some nurses since you are intern, she doesn't give you all the information regarding the patients, she gives you information about general small things. Whereas she gives other nurses more information, even the smallest details" - (SM). In addition, the ignorance of physicians was also identified as a challenge: "The doctors there ignore me and give orders without discussing them with me, even when I am in front of them in the patient's room" - (HM). The participants from the teaching hospital highlighted the unfair practice of nursing staff allocating difficult patients to nursing interns: "I was assigned the same patient for couple of days, then I figured out that the patient gives many orders and they had got bored of her orders" - (WT). In addition, some of the foreign nursing staff used their native language to communicate patient information during patient handover, which affected the interns' ability to understand the most important information regarding their assigned patients: "For the handovers I come early in the morning so I can see them, but if it is two Indian nurses I really don't understand, because they speak their own language"- (ET). Nevertheless, the nursing interns in the specialized research hospital stated that both nursing interns and staff were given a voice to discuss and argue patients' cases with physicians: "The nurses there really have a voice, they argue with consultants and physicians, not like in other hospitals"-(RS).

\subsubsection{Non-nursing Work}

The interns from both the military and teaching hospitals identified the significant challenge of being asked to do nonnursing work such as collecting medications from the pharmacy, sending patients for MRI or ECT scans, and working as a babysitter: "...pushing the patient or taking a medication from the pharmacy or taking a patient sample to the laboratory" -(SM); "I remember one called me by the bell just to stop her child from screaming and crying" - (ST).

\subsubsection{Time Management}

Time management was experienced as a challenge only in the specialized hospital, as the interns had to manage different elements such as patient care, attending workshops, and working on their checklist, which resulted in feelings of stress: "The time management, in the beginning I had so much to do, such as the patient checklist and workshops. It was difficult and so much stress" - (SS).

\section{Discussion}

This study investigated nursing interns' experiences regarding the factors and challenges affecting their clinical practice during their internship programme. It found that the hospital orientation programme was perceived by the nursing interns as a significant factor that facilitated their clinical practice. Moreover, the orientation provided by clinical instructors in some of the clinical units was also highlighted by the interns as an essential factor facilitating their clinical 
practice. This result corroborates the findings of a previous study which demonstrated that delivering an adequate orientation is the responsibility of both the clinical setting and preceptors [2]. This finding can be explained by the fact that hospital orientation is conducted to explain the main hospital policies and procedures and to make interns aware of the healthcare system adopted within the hospital. Additionally, hospital orientation was considered by all the interns as a foundation with which to establish best practice within their assigned clinical units.

The most significant finding that emerged from the analysis was that educational programmes designed specifically for interns were a critical factor affecting the participants' ability to gain the required knowledge. Additionally, it was interesting to note that mentorship programmes provided for the interns at the start of the internship period were also highlighted as a facilitating factor of their clinical practice. This result supports the finding of previous studies which reported that education and training are important in clinical practice [15], and that mentorship programmes are crucial to supporting junior nurses in their nursing practice and facilitating their professional development [16]. This finding is likely related to the importance of developing interns' knowledge, skills, and attitudes as well their clinical confidence to practice safely, and also the importance of providing nursing interns with an opportunity to experience a realistic working environment as a nurse in an employment situation in a healthcare setting and giving insight into the practice of professional nursing. In the current study, all interns described being involved in nonnursing work, such as being asked to do porter duties. This finding is consistent with a previous study which found that nurses in both medical and surgical units tend to be involved in non-nursing activities, especially in-patient transport and clerical work [17]. This is likely due to factors such as staff shortages within hospitals as well as unclear responsibilities for the nursing interns regarding their practice, which limited their opportunities to practice nursing procedures safely. Moreover, this study revealed that the nursing interns from some hospitals experienced unfair treatment from other healthcare professionals such as nursing staff and physicians. This finding is consistent with previous studies that have highlighted the unfair treatment and ignorance enacted by nursing preceptors, managers, and physicians towards nursing interns in clinical practice [4]. This finding is likely due to the fact that the nursing staff in the hospitals investigated tended to use the interns to deal with Arabic patients who had difficult personalities and attitudes. Additionally, the majority of healthcare systems in Saudi Arabia do not have a nursing voice; rather, the nursing staff follow physicians' orders.

\section{Conclusion}

Educational programmes, hospital orientation programmes, and mentorship programmes were highlighted as the most important facilitating factors for the interns' clinical practice. On the other hand, unfair treatment, the ignorance of healthcare professionals, and being involved in non-nursing work were all identified as challenging factors that negatively affected the interns' clinical practice.

\section{Recommendations}

This study revealed a need for continuous educational programmes to be provided for nursing interns by hospital education departments. In addition, it is recommended that nursing interns are allocated mentors at the beginning of their internship year through the provision of mentorship programmes by the hospital. A clear job description and clarification of responsibilities should also be provided for all interns so they will not be involved in non-nursing work. Furthermore, further research is needed to assess nursing interns' preparedness to work as nursing staff.

\section{Author Contributions}

All authors contributed to this work equally. S. Althaqafi, H. Alhatimi, A. Almalki, O. Alyamani, and M Banakhar were all involved in the process of data collection and analysis. All authors contributed extensively to the work presented in this manuscript. All authors contributed to writing up all the sections of the research project. Dr Banakhar supervised the project, gave feedback on the research at all stages, and reviewed the manuscript.

\section{Conflict of Interest}

The authors declare that there is no conflict of interest.

\section{Acknowledgements}

First and foremost, we thank our God, Almighty for helping us and enabling us to carry out this work. Then we would like to express our deep and sincere gratitude to our supervisor, Dr Maram Banakhar, for her vast knowledge, kindness, patience, encouragement, and continuous help and guidance. Also, we are deeply thankful to our participants. We want to acknowledge and appreciate their assistance and transparency during our research, as their information helped us to complete this study. Finally, we are extremely grateful to our parents for always being there for us, for their love, support, prayers, caring, and continuous encouragement.

\section{References}

[1] Saudi Commission for Health Specialities. (2019 Retrieved from https://www.scfhs.org.sa/Registration/Regulation/Pages/Nursi ng.aspx.

[2] Diane M. Haleem, Wendy Manetti, \& Kathleen Evanina, \& Robin Gallagher (2011). A Senior Internship Facilitating the Transition to Nursing Practice. Nurse Educator, Vol. 36, No. 5, pp. 208-213. 
[3] Olson, R. K., Gresley, R. S., \& Heater, B. S. (1984). The Effects of an Undergraduate Clinical Internshipon the SelfConcept and Professional Role Mastery of Baccalaureate Nursing Students. Journal of Nursing Education, 1984; 23 (3); $105-108$.

[4] Najjar, H. A., \& Rawas, H. (2018), Factors Affecting the Clinical Practice of Nursing Interns at Tertiary Hospital Jeddah in Saudi Arabia. International Journal of Studies in Nursing; Vol. 3, No. 3; 2018.

[5] Cheraghi, M. A., Salasli, M., \& Ahmadi, F. (2007). Iranian nurses' perceptions of theoretical knowledge transfer into clinical practice; A grounded theory approach. Nursing and Health Sciences, 9, 212-220.

[6] Edwards, H., Smith, S., Courtney, M., Finlayson, K., \& Chapman, H. (2004). The impact of clinical placement location on nursing students' competence and preparedness for practice. Nursing Education Today, 24 (4) 248-255.

[7] Atakro, C., Armah, E., Menlah, A., Garti, I., Addo, S., Adatara, P., \& Boni, G. (2019). Clinical placement experiences by undergraduate nursing students in selected teaching hospitals in Ghana. BMC Nursing (2019) 18; 1.

[8] Al-Momani, M. M. (2017). Difficulties Encountered by FinalYear Male Nursing Students in Their Internship Programmes. Malays J Med Sci. 2017 Aug; 24 (4); 30-38.

[9] Aboshaiqah, A., Tumala, R., Patalagsa, J., Al-Khaibary, A., Al Fozan, H., \& Ben, J. (2018). Perceptions of confidence among Saudi nursing interns during internship program; A crosssectional study. Ann Saudi Med. 2018 Jul-Aug; 38 (4); 288-298.
[10] Aboshaiqah, A., \& Qasim. A. (2018), Nursing interns' perception of clinical competence upon completion of preceptorship experience in Saudi Arabia. UK; Elsevier Volume 68, September 2018, Pages 53-60.

[11] Gray, J., Grove, s. \& Sutherland, S. (2017). The Practice of Nursing Research; Appraisal, Synthesis, and Generation of Evidence (8th ed.). UK; Elsevier.

[12] Polit, D. and Beck, C. (2018). Essentials of Nursing Research; Appraising Evidence for Nursing Practice (9th ed.). USA; Wolters Kluwer.

[13] Bryman, A. (2008). Social research methods (3rd ed.). New York, Oxford; University Press Inc.

[14] Goodman, C. \& Evans, C. (2010). Focus Group (6th ed.). Oxford; A John Wiley \& Sons Ltd Publication.

[15] Logina, A., \& Traynor. M. (2019). The relationship between effective transition models and the optimal management of patient care. British Journal of Nursing, 2019, Vol 28, No 3 .

[16] Weng, R., Huang, C., Tsai, W., Chang, L., Lin, S., \& Lee, M. (2010). Exploring the impact of mentoring functions on job satisfaction and organizational commitment of new staff nurses. BMC Health Services Research 2010, 10; 240.

[17] Al-Kandari, F., \& Thomas, D. (2008). Adverse nurse outcomes; Correlation to nurses' workload, staffing, and shift rotation in Kuwaiti hospitals. UK; Elsevier. Volume 21, Issue 3, August 2008, Pages 139-146. 